\title{
Cognitive pragmatics: The mental processes of communication
}

\author{
BRUNO G. BARA
}

\section{Introduction}

The basic idea behind this work is that communication is essentially a cooperative activity between two or more people in which the meanings of each transaction are constructed by all those actors together engaged in the shared task of reciprocally attending to the other communicants' words. The aims of the actors engaged in an interaction may differ, but to be able to say that communication has taken place successfully, all the participants must share a set of mental states. The responsibility for communication falls on the shoulders of each and every participant, for they act together in order to realize communication. Focusing on stereotypical roles such as speaker and hearer, or emitter and receiver, confounds the issue by fragmenting into isolated blocks, an activity that acquires meaning precisely from being global and collective. Moreover, I shall claim that human beings, unique among animals, possess a basic communicative competence that sustains both the linguistic and the extralinguistic way of expressing it. Furthermore, if we are to speak of communication and not simply of information transmission, then agents must devote themselves intentionally to such common activity.

The cognitive emphasis that can be seen in the titles both of the paper and of the book is justified by the fact that communication will not be examined here from the viewpoint of an external observer, as happens in linguistics and in the philosophy of language, where attention is focused on the finished product, whether this be an utterance or a discourse. Instead, I will take a standpoint within the mind of the individual participants, trying to explain how each communication act is generated mentally-before being realized physically - and then comprehended mentally by the other interlocutors. In the text, by the term "cognitive pragmatics" I shall refer to the theory presented in the following and to the related research program.

My intention is to describe the mental states of the participants in a communicative interaction. In addition to an analytical description, I will also furnish a formal definition of the various mental states, such as belief and intention, 
offering a number of innovations compared to traditional treatments, thereby offering solutions to problems that have hitherto not found satisfactory explanations. The mental states introduced will then come to constitute a logical model that accounts for both the production and the comprehension of communication acts in the ongoing process of their construction.

With regard to individual development, the central point is to define the stages in the acquisition of pragmatic competence, from the first few seconds of birth to adulthood. Hence, sets of experimental data taken from the literature and from my own work will be presented to support cognitive pragmatics. A valid theory should be able to correlate with the neurosciences, predicting how communicative capacities may decline with age, or suffer damage as a consequence of given pathologies that may be general — as in Alzheimer's diseaseor focal — as is the case with brain injury. I will support my theory with data from neuropragmatics and from brain imaging studies.

\subsection{Linguistic and extralinguistic communication}

The issues raised with regard to the differences between verbal and nonverbal behavior have been historically imprecise rather than controversial, for the distinctions that have been made are, first, based on intuitions and, second, contradictory. The difference between the two forms is founded principally on the nature of the input: Spoken language is defined as verbal, as is generally the case also with written language. Nonverbal language refers to all other forms: posture, facial expressions, gestures, space (between conversants), and time (between two following communication acts) (Hinde 1972).

This distinction creates many problems, all of which are substantial. The most serious is that the nonverbal category includes structured languages such as those for the deaf (for instance, ASL, American Sign Language). Other issues also produce devastating criticisms, highlighting the weakness of input as a distinguishing criterion: Why should the visual medium be assigned to the verbal category in the case of writing and to the nonverbal category in the case of observed behavior or of gestures? To which category should Braille, the language of the blind, be assigned? Since it is written, it should be classified as verbal; however, since it exploits tactile medium, it may be equally correctly categorized as nonverbal. In general terms, placing so much weight on the acoustic medium thus seems excessive; in particular, this excessiveness is underscored by the fact that the criterion is not applied in absolute terms: Prosody, which incontestably employs the voice, is classified as nonverbal.

I will therefore advance an alternative to the distinction based on input that is based instead on the way data are processed: linguistic communication is based on the communicative use of a system of symbols, whereas extralinguistic communication consists of the use of a set of symbols (Bara and Tirassa 
2010). Intuitively, the essential difference lies in the principle of compositionality: Language may be subdivided into smaller constituent components bearing autonomous meaning, that is to say words, whereas extralinguistic communication comes about through the use of components that cannot be decomposed into smaller, autonomous units: A smiling face is a smiling face, and not the sum of many small parts each of which is smiling. I shall present brain-imaging evidence in favor of my distinction in section 2.4, after introducing the theoretical concepts in need.

\subsubsection{Linguistic communication: Compositional. Linguistic communica-} tion may be defined as the communicative use of a system of symbols. This means that language is an entity based on compositionality: Language is built up recursively from separate components that may be joined together (Chomsky 1957). Some linguistic expressions have an atomic structure; that is, they may not be subdivided into smaller, constituent units. Other expressions have a molecular structure; that is, they are composed of smaller constituents that may in their turn be either atomic or molecular. The semantic content of an expression - be it atomic or molecular-depends both on its global structure and on the semantic content of its constituents.

1.1.2. Extralinguistic communication: Associative. Passing on now to extralinguistic communication, this form of communication may be considered the communicative use of a set of symbols. This mode is essentially noncompositional; that is, it consists of parts and not of constituents. Extralinguistic signals are molecular blocks that cannot be decomposed any further, inasmuch as they are equipped with intrinsic, global significance. The parts do not possess atomic meanings into which they may be further subdivided. The pirouette performed by a ballerina is a pirouette performed by the entire body, and not a pirouette that is performed by the left leg plus a pirouette that is performed by the right leg plus the torsion of the trunk and so forth.

\section{Mental states for communication}

Human beings possess, at any given time, a series of mental states. These may be both emotional and cognitive, and both of these may be either conscious or unconscious. Here we will deal only with those states that are relevant for an understanding of the process of communication; that is, states that are causally relevant in interactions between humans. I omit some states on the assumption that they do not constitute primitive notions that form part of the process of the production and comprehension of communication acts. 
I analyze in the book the following states: attention, belief, knowledge, and consciousness. Volitional primitives are also dealt with: intentions, goals, plans, and motivations. Although all are important, I shall focus here only on the concepts of shared belief and of communicative intention.

\subsection{Shared belief}

The concepts of knowledge and belief are closely connected in the literature on pragmatics. Such concepts are standardly formalized as predicates or modal operators. Traditionally, belief is employed as a primitive. The properties of a belief are defined by a set of axioms derived from the theory of logic developed by Jaakko Hintikka $(1962,1966)$. Knowledge, by contrast, is a derived concept, that is, a sort of abbreviation of true beliefs about the world.

To introduce the concept of shared beliefs - a concept that is indispensable when we are dealing with mental states in communication-I must first differentiate between three types of beliefs: individual, common (also called mutual), and shared. I will do so in an intuitive fashion, allowing myself a certain definitional leeway.

In some cases the agents believed a certain thing, or believes that the other agents believes a certain thing, but in a totally autonomous fashion, with no connection existing between the agents themselves. We will call this type of belief individual belief.

Often, however, in a given context, all the agents have the same individual beliefs: All agents generally share knowledge of their surrounding environment, or a certain amount of knowledge that is culturally transmitted. For instance, A may share with B a love of opera, and, with all pacifists, the opinion that all atomic weapons should be banned, and, with all humans, the evidence that we are born of a mother and a father. Much human interaction is based on this type of belief, which is spread over a more or less wide group of people, and which we will call common belief or mutual belief.

Clark (1996) speaks of common ground, meaning the sum of knowledge, beliefs, and suppositions that two or more people share. Common ground enables us to identify a series of cultural communities, which may be classified according to the type of beliefs a community shares.

However, having common beliefs is not a sufficient condition enabling communication to take place. Suppose that a person is in a foreign country whose language she does not know, and she wishes to convey her mental state to other interlocutors: She will not employ a gesture whose meaning she is familiar with unless she thinks the other participants are also cognizant of its meaning. There is no point in making a gesture with which only she is familiar. At the outside, everyone present might happen to know the gesture at an individual level, and yet never use it because they are not aware that all the others are also 
aware of the meaning it conveys. The conclusion, therefore, is that in order to communicate, in addition to possessing common beliefs, each participant must also be aware of the fact that all the other participants possess those very same common beliefs.

I define a shared belief as that belief that is not only common to all the participants engaged in the speech event, but of which each participant is aware is possessed by all the other participants. From a psychological standpoint, shared belief has a crucial feature: It is subjective, and not objective, as is common belief. In actual fact, no one can ever be certain that another person has knowledge of a certain type: She may at most assume that he has it, and may be convinced that they share it. To be certain, or to have true knowledge, as Hintikka would say, she should in some way be able to observe the mental states of others in some direct manner, and not simply infer them from circumstances. In theory, I might pretend to share with others the belief that the earth is round, but be privately convinced that it is flat, without anyone ever suspecting what is really going on in my mind. Taking up a subjective position in which shared belief is concerned means, fundamentally, assuming that each agent has a space of shared beliefs that contains all the beliefs the agent herself is convinced she shares with a given partner, or with a group of people, or with humanity in its entirety.

The formal connection between belief and shared belief is established by the so-called fixpoint axiom (Harman 1977), which captures the circularity of mutual belief:

$$
\text { (1) } \mathrm{SH}_{A B} p \equiv \mathrm{BEL}_{A}\left(p \wedge \mathrm{SH}_{B A} p\right)
$$

where $\mathrm{SH}_{A B}$ means that both the agents $\mathrm{A}$ and $\mathrm{B}$ reciprocally hold the belief that $p$. What formula (1) expresses is that when actress A takes $p$ as shared by $\mathrm{B}$ and herself, this means that on the one hand she herself takes $p$ as being true, and that on the other hand she believes that B also takes $p$ as being shared by both of them. Circularity derives from the fact that sharedness is present on both sides of the formula, both in the definiens and in the definiendum. By distributing belief $\mathrm{BEL}_{A}$ on the conjunction, infinite implications of the following type may be obtained from formula (1):

(2) $\mathrm{SH}_{A B} p \supset \mathrm{BEL}_{A} p$

$\mathrm{SH}_{A B} p \supset \mathrm{BEL}_{A} p \mathrm{BEL}_{B} p$

$\mathrm{SH}_{A B} p \supset \mathrm{BEL}_{A} p \mathrm{BEL}_{B} p \mathrm{BEL}_{A} p$

$\mathrm{SH}_{A B} p \supset \ldots$

The symbol "ऽ" corresponds to the concept "implies." Formula (2) expresses the possibility of generating a theoretically infinite sequence of individual beliefs, starting from a shared belief. The following constitutes a more intuitive translation: 


\section{AUTHOR'S COPY | AUTORENEXEMPLAR}

(3) Since in A's opinion, she and B share the belief that $p$, this implies that:

A believes that $p$.

A believes that $\mathrm{B}$ believes that $p$.

A believes that $\mathrm{B}$ believes that $\mathrm{A}$ believes that $p$.

... and so on, potentially ad infinitum.

One important difference between mutual belief and shared belief (1) is that the former is objectively common to both interlocutors. This means that both A and $\mathrm{B}$ really do believe that $p$, and both should therefore possess the same mental state corresponding to the belief that $p$.

Shared belief, on the contrary, assumes a subjective viewpoint, since no agent can ever be sure that all the other participants possess the same beliefs she holds. Hence, shared belief always expresses the standpoint of one of the interlocutors. A may take a certain fact as shared by both B and herself, but this assumption is subjective, one that does not necessarily correspond to the real mental states possessed by B. No one can open another person's brain and look inside in order to check out what beliefs the other person actually does hold. And as we shall see in section 5.2, subjective assumptions regarding sharedness play an important part in nonstandard communication, especially in cases of irony and deceit.

\subsection{Communicative intention}

Let us now examine what happens when instead of speaking of action in general we focus our attention on communication acts. One fundamental difference between actions pure and simple and communication acts is that the latter are always carried out together with someone: Communication does not come about in isolation or in complete autonomy. Thus, though we may speak of a single agent when we refer to actions in general, when we enter the domain of communicative interaction we must always have at least one actor (A) and one partner (B) to whom the act is directed. Other agents (C, D, etc.) may participate as audience to the communicative event. I define communicative intention as the intention to communicate something, plus the intention that that intention to communicate that particular something be recognized as such. To be more precise, A possesses a communicative intention that $p$, with regard to $\mathrm{B}$ - that is to say: A intends to communicate that $p$ to $\mathrm{B}$ - when $\mathrm{A}$ intends the following two facts to be shared by both A and B:

1. that $p$

2. that $\mathrm{A}$ intends to communicate that $p$ to $\mathrm{B}$

However, making information achieve the status of sharedness does not mean one has communicated it. The necessary condition for real communica- 
tion to take place is that such information be intentionally and explicitly proposed to the interlocutor. Grice (1975) points out that communicating includes not only the speaker's first-order intention $I_{1}$, that of achieving a certain effect on the interlocutor, but also the second-order intention $I_{2}$, namely, that the firstorder intention $I_{1}$ be recognized as such by the interlocutor.

For example, by wearing my King's College tie, I make the fact that I belong to the teaching staff of that institution shared, but it cannot be asserted that I communicated this particular fact. The first-order intention $I_{1}$, namely, that of letting others know where I teach, has been satisfied, but the second-order intention $I_{2}$, namely, that other people recognize that I desire to communicate this fact, has not been satisfied. It would have been satisfied had I openly declared:

(4) I teach at King's College, Cambridge.

In this case, listeners would gain awareness not only of the specific fact, but also of my open desire that they become aware of that fact.

Formally, communicative intention may thus be defined as follows:

$$
\mathrm{CINT}_{A B} p \equiv \mathrm{INT}_{A} \mathrm{SH}_{B A}\left(p \wedge \mathrm{CINT}_{A B} p\right)
$$

What formula (5) means is that $\mathrm{A}$ has the communicative intention that $p$ toward $\mathrm{B}$ (in symbols, $\mathrm{CINT}_{A B} p$ ) when $\mathrm{A}$ intends $\left(\mathrm{INT}_{A}\right)$ that the following two facts be shared by $\mathrm{B}$ and herself $\left(\mathrm{SH}_{B A}\right)$ : that $p$, and that she intended to communicate to $\mathrm{B}$ that $p\left(\mathrm{CINT}_{A B} p\right)$.

All of this may be translated into more acceptable English as follows. A intends to communicate a certain thing to B. A concurrently desires that B take as shared between the two not only the specific content she wishes to convey, but also the fact that she actually did wish to convey that content to him.

Similar to shared belief, communicative intention is also a primitive in pragmatics. This means that it implies, but is not reducible to, an infinite number of finite embeddings of intentions and shared beliefs. The following logical implications may be derived from formula (5):

$$
\begin{aligned}
& \mathrm{CINT}_{A B} p \supset \mathrm{INT}_{A} \mathrm{SH}_{B A} p \\
& \mathrm{CINT}_{A B} p \supset \mathrm{INT}_{A} \mathrm{SH}_{B A} \mathrm{INT}_{A} \mathrm{SH}_{B A} p \\
& \mathrm{CINT}_{A B} p \supset \mathrm{INT}_{A} \mathrm{SH}_{B A} \mathrm{INT}_{A} \mathrm{SH}_{B A} \mathrm{INT}_{A} \mathrm{SH}_{B A} p \\
& \mathrm{CINT}_{A B} p \supset \ldots
\end{aligned}
$$

Formula (6) means that given the fact that $\mathrm{A}$ intends to communicate a certain thing to $\mathrm{B}$, we may infer that $\mathrm{A}$ also intends that her original intention to communicate that particular thing be recognized. If need be, this includes the further inference that A wishes B to recognize her intention of letting B know that she really did intend him to become aware of her intention to communicate to him that particular message. And so on, until the cognitive resources possessed by both manage to make sense of the sequence of embeddings. 


\subsection{Brain imaging evidence for communicative intention}

The brain-imaging studies I shall summarize offer converging evidence in favor of two basic tenets of cognitive pragmatics theory: that communicative intention has a special status in our brain/mind, and that communicative competence is modality-independent. In empirical science final validations do not exist (Popper 1934), but convincing experiments help.

2.3.1. A dynamic intentionality network. In order to understand what a communicative intention is, we have to classify the different types of intentions. The first distinction we need is provided by Searle (1983), and is that between prior intention and intention-in-action. Searle regards the intentionin-action (or motor intention) as the direct cause of an agent's movement: the mental and causal component of the bodily movement of an action. To cover the overall conditions of an action, one has to analyze also the prior intention, which orients the action as a whole. Prior intention is formed in advance, to represent goal states that may be quite distant from the chain of actions that lead to their fulfillment: prior intention represents the goal of the action before it is undertaken.

Figure 1 shows the three kind of prior intentions we have tested through fMRI.

Private intentions (PInt) require only the Actor to perform actions adequate to reach her goal (e.g., A intends to drink a glass of water). On the contrary, a social intention requires at least a Partner, who is necessary for the Actor to reach her goal (e.g., A intends to play tennis with B). In my approach, the prototypical case of a social intention shared in the present is Communicative intention (CInt).

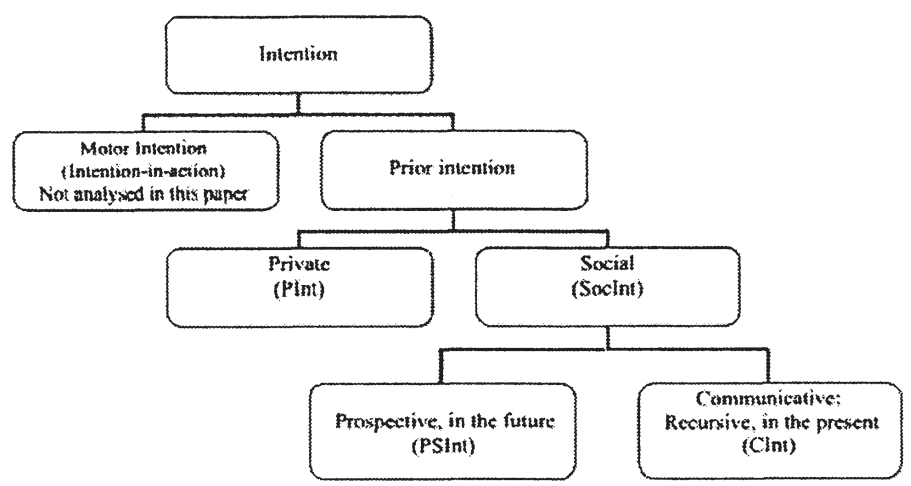

Figure 1. Classification of the different types of prior intention. 
However, there are special intentions whose social goal lies in the future. For instance, Alice may intend to visit her father next Sunday. This kind of social intention involves the representation of a social goal when A and B are not actually interacting but B is part of A's goal. We define this kind of intention Prospective social intention (PSInt). In the case of PSInt, the desired social interaction is not present at the moment, but if the PSInt will become shared in the future, then it will cause the social interaction to occur.

According to Bratman's (1987) account, the main characteristic of futuredirected intention (as he prefers to call prior intention) is that the Actor does not only want to accomplish that intention, but she is also committed to do it. If Alice forms the prior intention to visit her father next Sunday, she is committing herself now to visit him on Sunday. Pacherie and Haggard (2010) call all kinds of prior intentions prospective intentions, because they have to be realized in a future moment. For the sake of simplicity, in this paper I reserve the term "prospective intention" only to social ones (PSInt), even though in principle also private intentions may be future-directed, e.g., Alice may now form the prospective private intention to give up smoking on her next birthday.

In performing our fMRI experiments our main predictions were threefold: (1) the Medial PreFrontal Cortex (MPFC) areas involved in intention recognition are activated only in response to social stimuli requiring comprehension of social interaction, both present and future; (2) the posterior areas underlying intention recognition (right Temporo-Parietal Junctions (TPJ) and precuneus) might suffice for representing the mental states of agents, as long as those agents are acting outside social interaction; (3) the existence of a dynamic brain network encompassing the regions usually involved in mind reading, i.e., the right and left TPJ, the precuneus and the MPFC, each of which with a specific function depending on what type of prior intention is represented starting from an observed action.

A first series of experiments (Walter et al. 2004) allowed us to propose a dynamic intentionality network; a deeper level of analysis based on signal time courses for the four regions of interest extended and confirmed our earlier results (Ciaramidaro et al. 2007). In sum, our results show that whereas the right TPJ and the precuneus are necessary for processing all types of prior intentions, the left TPJ and the MPFC are specifically involved in the understanding of social prior intention. More specifically, the left TPJ is activated only when social prior intentions occur in the present: this is the case of communicative intention (Bara et al. 2011).

A subsequent step has been the testing of the intentional network not only on healthy individuals, but also on people with schizophrenia and with autism. We hypothesized that paranoid schizophrenics could show hyper-intentionality, i.e., the tendency to over-attribute intention not only to persons but also to objects. The thesis has been confirmed in a group of 12 patients with paranoid 


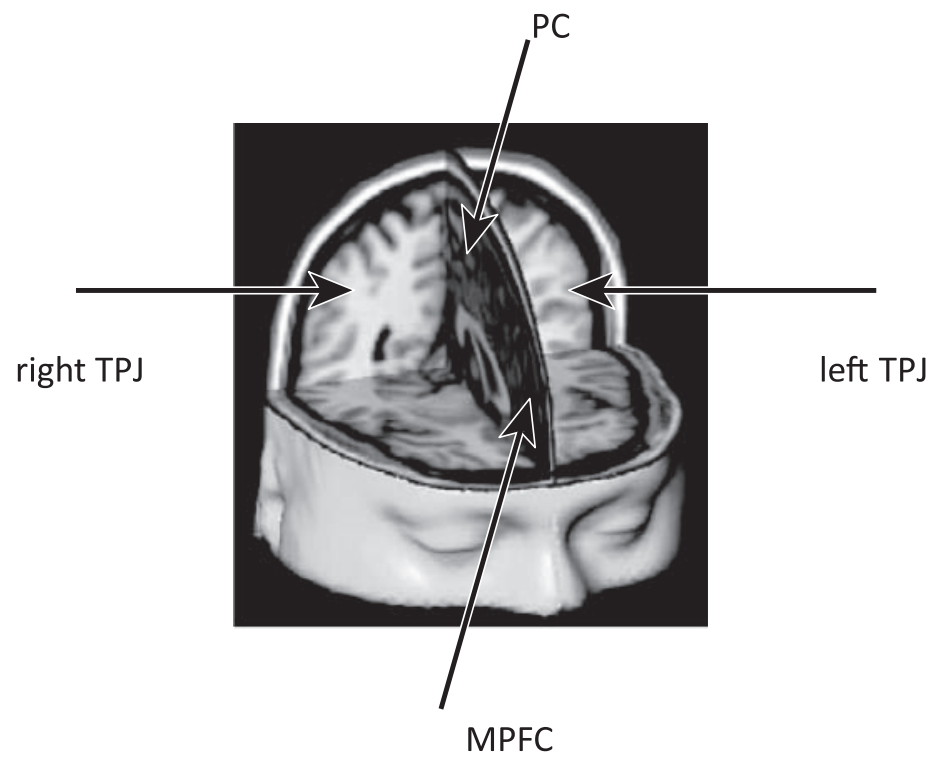

Figure 2. The intentionality network including the right and the left temporo-parietal junctions $(T P J)$, the precuneus $(P C)$, and the anterior paracingulate cortex ( $(P C C)$, located in the medial prefrontal cortex (MPFC).

schizophrenia: their intention detector became hyperactive in the paranoid interpretation of the physical world (Walter et al. 2009).

Adopting a line of reasoning similar to Crespi and Badcock (2008), we claim that the impairment in understanding others' intentions exhibited by paranoid patients and autistic patients can be considered as the two extremes of a continuum. People with autism are hypo-intentional, as they tend not to attribute the features of sociality to actors neither interacting between them (CInt), nor preparing a social interaction (PSInt). The autistic comprehension of the social interaction is quite similar to their interpretation of the physical world (Bara et al. 2011).

\subsection{Brain imaging explorations of linguistic and extralinguistic modalities of communication}

As explained in section 1.1, I assume that a common communicative competence - independent of the linguistic or extralinguistic gestural meansis instantiated at the level in which a communicative intention is inferred and comprehended within a specific social context, that is, at the pragmatic level. Consequently, I hypothesize that there is no difference in brain activity be- 
tween the recognition of a communicative intention issued by an observed linguistic behavior and the recognition of the same communicative intention issued by an observed gestural behavior: The dynamic intentional network should be recruited independently of the modality used to convey the different intentions.

Enrici et al. (2011) in a fMRI study on 24 healthy participants showed that linguistic and gestural modalities share a common communicative competence at the pragmatic level, viz. at the level at which an actor's communicative intention has to be reconstructed by a partner. The second issue we wished to address was whether any additional brain areas other than those involved at the pragmatic level, that is, the intentionality network areas, are specifically recruited depending on the modality used. Perisylvian language areas are recruited by the linguistic modality, whereas sensorimotor and premotor areas are recruited by the extralinguistic modality. In conclusion, we proved that the intentionality network is modality independent, while the expressive means involved activate the brain regions corresponding to language or to gestures.

\section{Behavior games: The social framework of communication}

In conversation, we must make a clear distinction between communicative competence and interaction schemas. Communicative competence is a general characteristic of the mind, whereas stereotypical interaction schemas are culture-bound. Indeed, the latter may pertain to a small group of individuals, or even two people. The idea that will be developed in this chapter is that communicative competence may be viewed in formal terms as a metalevel property that controls first-level inferences; such inferences are carried out on shared representations of stereotypical interaction schemas.

Consider the following concrete example:

(7) A: Tomorrow's Thursday. Will you coordinate the exam supervision?

B: Actually, the Vice Chancellor has fixed a meeting for 9 a.m.

In every standard context, B's reply would be taken as a justification for refusing to carry out A's request. As stated earlier, B cooperates conversationally but not behaviorally. The intuitive concept of a behavior game allows us to explain conversation (7), for it enables us to assert that, through her request, $\mathrm{A}$ is proposing that she and $\mathrm{B}$ play the behavior game:

(8) Pedagogical Duties

A is responsible for running the departmental activities from Monday to

Wednesday;

$\mathrm{B}$ is responsible for running the departmental activities from Thursday to Saturday. 
With his reply, B rejects A's proposal, justifying himself by explaining he has a duty that takes priority over examinations. A thus takes B's reply as concurrently constituting a counterrequest on B's part to take over his exam session. The point is that in order to cooperate, at least at the level of conversation, both agents must share the behavior game Pedagogical Duties. In real terms, mutual knowledge of game (8) is exploited to achieve conversational cooperation, even if B fails to execute the moves foreseen by A, and, therefore, behavioral cooperation is not achieved.

The reason for introducing behavior games is that the literal meaning of an utterance is only the departure point for its comprehension. "Why is she saying this to me?" and "What does she want from me?" are the real questions requiring an answer. If someone sitting in the reader's office says to him:

"I'd like a coffee."

it is obvious that she is proposing a game such as Hospitality, and the rules of that particular game place the encumbrance of providing coffee on the host. And indeed, either the host does produce coffee or else he is obliged to explain why he has not deigned to satisfy the indirect request. If the same assertion were to be uttered in a context in which the reader is not the person responsible for the pleasurable aspects of the situation, for example in the course of taking a walk in the park with a colleague, then that utterance will be interpreted as a proposal to play a different behavior game, for instance Going to the Cafe. However, if a stranger pops his head round the reader's office door and utters (9), then the poor reader will be utterly at a loss as to how to interpret that utterance. In real terms, either the reader is able to find a behavior game that will enable him to interpret the statement, in which case he will know what the stranger expects of him, or else he will be nonplussed.

Although the semantic meaning of expression (9) is immediately comprehensible, the effects the speaker wishes to activate in the hearer must be induced. In the case just mentioned, there is no context that enables both the reader and the stranger to identify a behavior game known to both parties that may be connected to the utterance: hence the failure to understand.

A behavior game is that structure which enables actors to coordinate their interpersonal actions, and that actors employ to select the intended meaning of an utterance among the many meanings that utterance might in theory convey.

\subsection{The structure of a behavior game}

For two actors to cooperate at the level of behavior, they must operate on the basis of a plan that is shared at least in part. Following Airenti, Bara, and Co- 
lombetti (1993), I will call a behavior game between A and B an action plan that is shared by A and B. The shared knowledge required for two actors to be able to interact in the same game may be a combination of tacit and explicit. As we shall see later, the two actors may have an explicit representation of the game, or they may have a tacit representation that is sufficient to enable them to direct their actions. Stated differently, for a game to be playable, it must be represented in the actor's memory.

In addition to actions, behavior games include validity conditions that specify the conditions under which the game may be played. Validity conditions may be viewed as an extension of the felicity conditions that Austin (1962) invoked to guarantee the success of performatives. The essential features of validity conditions, which do not apply only to performatives but to any move of the game, are time and place. However, some games may impose other conditions connected to the mental states of the participants, or constrained by the actions to be executed.

Finally, a game is playable only if the relationship between the participants allows it. In some cases, if the game has wide social applicability, as is the case with someone asking the time, then the participants need not even be acquainted. In other cases, participants must be members of the same group. For example, only two freemasons who have both recognized their common status as freemasons may discuss topics reserved to those belonging to that lodge. At the extreme of this type of case we have games that may be played only by two specific players and by no one else. This is the case, for example, with games played by parent and child, or by a married couple.

The relationship is therefore the set of behavior game that two people may play together.

The structure of a behavior game is shown in Figure 3. The first thing to note is that each agent has her own subjective, and not objective, vision of the game, since the entire process is based on the notion of shared knowledge, this latter notion also being subjective and not objective. In our notation, then, beside the name of each game there should appear the perspective of each player: $G$ (A, B) represents game $G$ viewed from A's standpoint, while $G(B, A)$, instead, represents the same game, G, viewed from B's standpoint.

We will now examine some examples to clarify the concept of behavior game. The first instantiation is one that is widely applicable, the game that allows us to ask a stranger the way.

Assigning games a declarative knowledge structure means that we can talk about them, mentioning them if needs be. This does not imply, however, that the actors in a game must be aware of the structure of the game, of the moves, or of the motivations that induce them to play the game. In other words, players may have, but need not have, an explicit representation of the games they play. 


\section{[NAME OF THE GAME]}

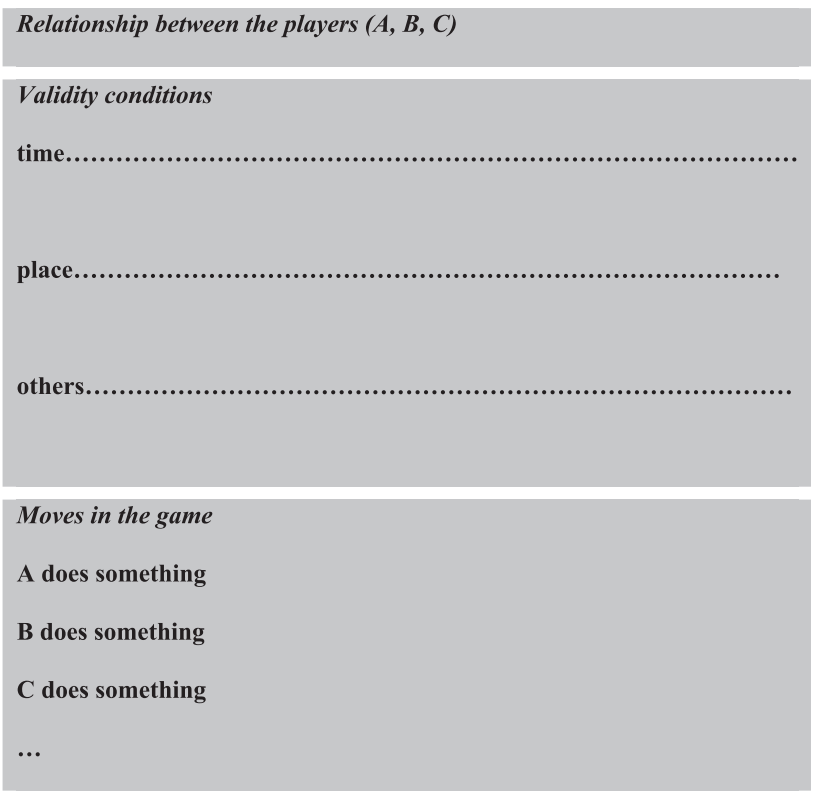

Figure 3. Structure of the behavior game.

\subsection{Types of game}

Among the various criteria that may be used to classify the different types of behavior games, I will, in the first instance, privilege an extensional criterion. I will consider games as having essentially an equivalent structure, differing with regard to the number of people capable of playing them.

There are three main types of games: Cultural games are common to an entire culture, affecting a large number of people. Group games are shared by a more or less restricted number of people forming a given group. Couple games are shared by only two people.

We will now examine these three types singly, bearing in mind that a classification of this nature is not based on rigid, watertight distinctions (Figure 4).

3.2.1. Cultural games. "Culture" as I intend it refers to large-scale phenomena that may vary from the civilized world to Western culture, from the Mitteleuropa tradition to the Parisian style. What this means is that if two people belonging to the same culture find themselves in a given situation and a specific game is enacted, both know reciprocally what they expect the other to do. 


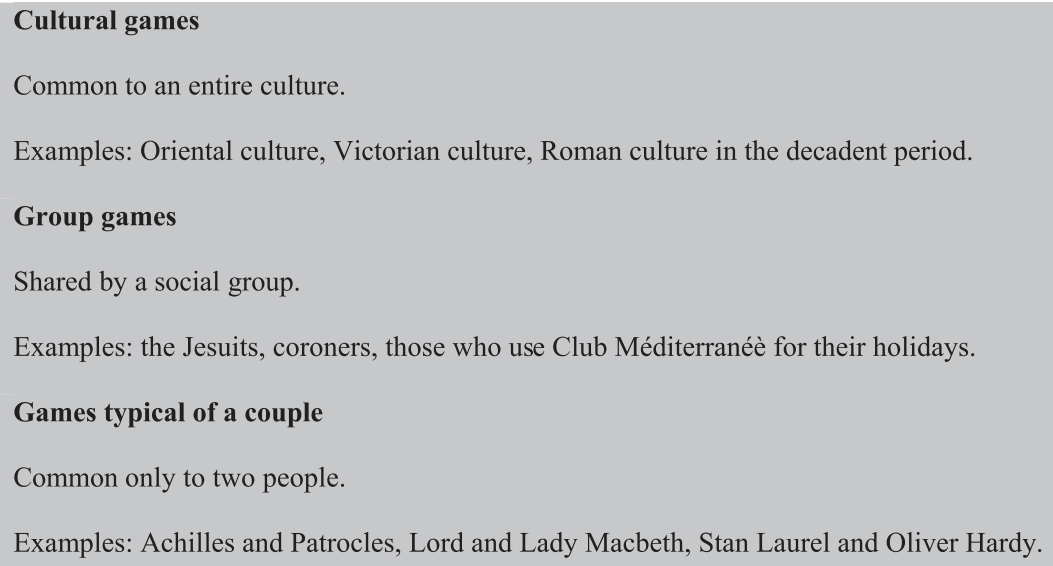

Figure 4. Game types.

Naturally, the more the game is widespread, the more the expected behavior approximates to a social norm. As such, it may be taught explicitly as well as through experience. Examples range from asking someone for directions to the multiplicity of rites laid down by books on etiquette. That such rules change from one country to another is something the traveler is well aware of: Saying one is sorry is as obligatory in the West as it is forbidden in Japan, for in the latter country excusing oneself would be interpreted as a refusal to take responsibility for one's actions.

Studies on intercultural communication (Piller 2010) and on linguistic anthropology (Duranti 1997) have shown that culture affects the different pragmatic possibilities of realizing interaction. Also due to the influence of crosscultural psychology (Berry, Poortinga, and Pandej 1997), this approach has caused the weakening of the thesis of universality and a greater attention to an ethnocentric dimension. As examples we can think here of the deployment of compliments and expressions of thanks. These are rigidly defined by each culture, but their workings are far from obvious. For example, table manners vary from belching at the end of the meal to serving oneself to each dish twice and twice only (the first time to be courteous, the second time to show one has appreciated the food).

According to cultural psychologists, common ground between people consists in large part of a repertoire that is "in the mind" because it has been put there by the communities into which the individual was born, has lived in, and in which he now lives (Tomasello 2009). 
3.2.2. Group games. In the case of a group game, the game is shared by a more-or-less restricted circle of people, and these people have normally shared the experience of structuring the game themselves. Examples of groups of this nature include Harvard Law School graduates, the Sicilian mafia, and Vietnam War veterans.

3.2.3. Couple games. Couple games are played by two people and are valid only for those two particular people. The game played by a couple may be extended into a group game. Typically, two friends, two colleagues, or a married couple may create the new game's structure.

Groups personalize cultural games, whereas couples personalize both cultural games and group games, constructing recognizable variants. For instance, the way two friends greet each other may turn into a playful insult instead of the usual exchange of well-wishes. It makes use of exactly the same mechanism by which a dialect of a subgroup is formed from the national standard language.

One might ask if wider games exist than those described above, a kind of set of universal games, applicable to humankind as a whole. There certainly exist behavioral schemas common to all human beings - think, for example, of a mother protecting her young who is looking for care-but to be such they must be genetically determined, hardwired into our brain right from birth. It is precisely for this reason that behaviors of this type do not come within our definition of a game. A game remains a structure that must be learned, one that may be negotiated and rejected. For this reason, if some behavioral pattern is innate, it cannot be considered a behavior game. Of course, any specific action, innate or learned, may be utilized as a move within a game.

\subsection{Playing a game}

An actor will play a game, provided two conditions come about: that the game be playable, and that the actor is interested in playing the game. Once the validity conditions are guaranteed, a game must be negotiated, that is, it must be proposed and accepted by all those who commit themselves to taking part in it. Once the game has begun, it will be played by the participants for the entire course of its logical lifespan, until it reaches its natural conclusion.

3.3.1. Bidding. Bidding may take the form of a communication act or of a behavioral act corresponding to the first significant move in the game. The communication act mentions the game itself, either by name or as a metonym, that is, by referring to any of the constituent parts of the game, for instance by mentioning one of the validity conditions or one of the moves.

Once the game has been opened, it remains active until it is formally closed. Generally, it is the players themselves who indicate that the game they priorly 
agreed to play is to continue through their behavior. Not all the moves made need to be attributed to the game, but when games continue for a long time, the players ensure they periodically confirm the meaning to be attributed to their actions.

In the case of competitive games, the participants must be satisfied with the way the procedures have been carried out, seeing that both parties cannot be satisfied with the results. The concept of "knowing how to lose" refers to respecting "fair play" even in defeat. This concept is greatly valued in highly competitive environments, where interpersonal relationships must survive both victory and defeat. My daughter Helen bursts into tears every time she loses at cards, taking offense in equal proportion at fate and at the winner: At 10 years of age, she has not yet developed the concept of making do with the happiness provided by the procedure, given that it is difficult to always be happy at the result.

3.3.2. Moves. A behavior game specifies the moves that constitute that game, at the highest level of detail possible in order to avoid obliging the actors to employ one sole mode of execution.

Broadly speaking, participants must agree as to whether a specific realization of a move may be held valid with respect to the context and the players' goals. If a move in the game lays down that something must be done that will please one's partner, then the action chosen by the actor will depend on what she believes will make her partner happy on that given occasion. A Havana cigar, a bottle of port, two tickets to the concert or to Polynesia: anything will do, provided it is appropriate to the situation.

\subsection{Breaking of a game}

Having begun a game does not necessarily mean one has to finish that game. Nevertheless, an actor who withdraws from a game is subject to social sanctions whose severity is proportionate to the importance the game has for the community. Breaking off a game does not include a person's desire not to play that game in the first place, an option that is always open to a person. Instead, the term refers to an actor beginning a game and then withdrawing when it is her turn to make a given move.

Sometimes it is the law that guarantees a contract will be respected. In other cases, it is the social group that penalizes the individual who does not respect the rules by attempting to avoid the commitments taken on.

The essence of social penalization is being considered untrustworthy. This is why in many cultures "losing one's honor" is so often associated with death, either at one's own hand or at the hand of another. The punishment for not having kept one's word is therefore always significant, and correlated with the value that the given commitment has in that cultural context. 
From an evolutionistic point of view, the cooperative style typical of human beings obliges the group to discourage free-riders, those who benefit from participating in mutualistic endeavors without doing their part. In addition to the bad reputation that a free rider earns for himself, and which makes him a second-choice partner for the others, the honest members of the group are ready to sacrifice a part of their resources for the satisfaction of seeing him punished. Tomasello (2009) claims that both norms of cooperation and conformity are cemented by guilt and shame ("I judge myself badly before others do, if I misbehave"). I doubt that critical self-judgment be a universal human trait, but together with ill fame and threats of punishment it definitely strengthens social norms and institutional respect.

\subsection{The relationship between the players}

For two players to be in a position to play a game, the first issue to be cleared up is whether the nature of the relationship between them is of the type that will enable them to interact through engaging in that particular game. One static definition of the relationship between two people consists in listing the types of games they mutually recognize they may play together.

Cultural games are open to all those who share the same culture: It is generally sufficient that both people speak the same language to ensure that they can both adequately play a game of this type.

For group games to constitute part of the games two players may engage in together, both partners must recognize the condition that they belong to the same group. Indeed, the initial stage of an encounter between two strangers is usually devoted to determining whether there are groups that both people belong to:

(10) Are we by chance both linguists, or vegetarians, or supporters of Milan Football Club, or card-carrying members of the Republican Party, or opera fanatics, or ex-convicts, or both divorced?

The set of groups the two people are both members of defines the type of games that will be mutually recognized, even though it does not automatically follow that a game that is known will have to be played. Group membership always creates some degree of reciprocity among people.

Furthermore, as we have already seen, acquaintance with a game does not imply that both people will wish to engage in that game with each other. This emerges in an even starker fashion when we consider games played by couples, which are connected to two specific individuals. We may be willing to accept physical contact with a friend though we find such behavior in others intolerable.

The relationship thus becomes the generator of constraints and affordances, starting from games that have been played or that unquestionably belong to the sphere of common culture, to arrive at those games that may potentially be 
played, but whose execution depends on the reciprocal perception of the two actors. Thus, in asking the other person something, the actor implies that she believes that both parties consider the relationship such that it justifies the question she has just asked.

\subsection{Conversation game}

A conversation game may be defined as a set of tasks that each participant in the conversation has to fulfill in a given sequence. Each task is characteristic of a phase in the generation/comprehension process that will be described in the next session. Furthermore, a conversation game specifies how the different phases have to be linked up both in standard and in nonstandard cases.

In each phase, the task associated with that phase is carried out employing a set of inference rules called base-level rules. The conversation game may be epitomized as a set of metarules that define both the task to be carried out in each phase and which task is to be activated next. The concept of a rule is employed in formalizing an analytical description. Naturally, nothing of the sort exists in the brain. Everything is realized through neuronal activity.

In each phase, the metarule associated with that phase defines the task by means of a logical formula that is obtained from the application of the baselevel rules. In addition, the metarule also establishes what must be done both when the task is completed and when it is not executed.

Thus it is the conversation game that establishes how a dialogue (a sequence of speech acts) is carried out. A dialogue is a highly structured activity involving at least two agents.

Cognitive pragmatics views the global structure of dialogues as deriving from sharing the knowledge of an action plan. Consequently, the global structure of a dialogue does not derive from linguistic rules, but from behavior games. In sum, the behavior game governs the interaction as a whole, whereas the conversation game is responsible for the harmonious local development of the dialogue.

\section{Generation and comprehension of communication acts}

I do not intend to analyze an elementary exchange in a dialogue in formal terms, but to explain the scheme of conversation in its entirety, since the latter is a special and fundamental case of communication among humans. Conversation is not so much a game of table tennis, in which the agents alternatively exchange information, as a communal and simultaneous effort to build something together.

Awareness of this fact does not, however, exempt us from analyzing conversation down to its smallest unit, for a microscopic decomposition of the com- 
munal construction brings out the alternating nature of the dialogic structure, a structure that is also composed of intervals between the recognizable alternating turns. The construction of meaning takes place in parallel fashion, not consecutively. Analysis, however, has its own set of requirements, and decomposition into constituent units is just as useful as the reconstruction of the full complexity of the natural state of conversation.

The general scheme is as follows: the actor produces an utterance; the partner builds a representation of its meaning. The mental states of the partner pertaining to the topic of the conversation may be modified by comprehension of the utterance(s). The partner then plans the next move in the conversation, which he then generates. The rules proposed comprise a dyadic model of communication acts that range from comprehension to reaction, that is, from the reconstruction of the meaning intended by the speaker to the establishment of the high-level intentions required to generate the response.

Assuming that actor A produces an utterance addressed to partner B, we may distinguish five logically connected steps in B's mental processes:

Stage 1. Expression act, where A's mental state is reconstructed by B starting from the locutionary act.

Stage 2. Speaker meaning, where B reconstructs A's communicative intentions, including the case of indirect speech.

Stage 3. Communicative effect, which consists of two processes:

(a) attribution, where B attributes to A private mental states such as beliefs and intentions; and

(b) adjustment, where B's mental states concerning the topic of the conversation may be altered as a result of A's utterance.

Stage 4. Reaction, where B produces the intentions he will communicate in his response.

Stage 5. Response, in which B produces an overt communicative response.

The linking together of these five stages is managed by the conversation game: stated more formally, it is the equivalent of a set of metarules. The standard sequence is that described, from stage 1 through to stage 5. However, if any one of the first three stages fails to complete its task, the normal chain is interrupted and the process moves directly on to the response stage. This is due to the fact that the conversation game lays down the rule that the partner will react to the actor's utterance, even when he does not understand it. This he may do, for instance, by asking for clarification. The global outline of these five stages is sketched out in Figure 5.

The execution of each task is governed by a set of base-level rules that define which dominion-dependent inferences are to be employed to carry out that task. These rules have different roles in the various processes. I must stress that 
Stage

Stage task

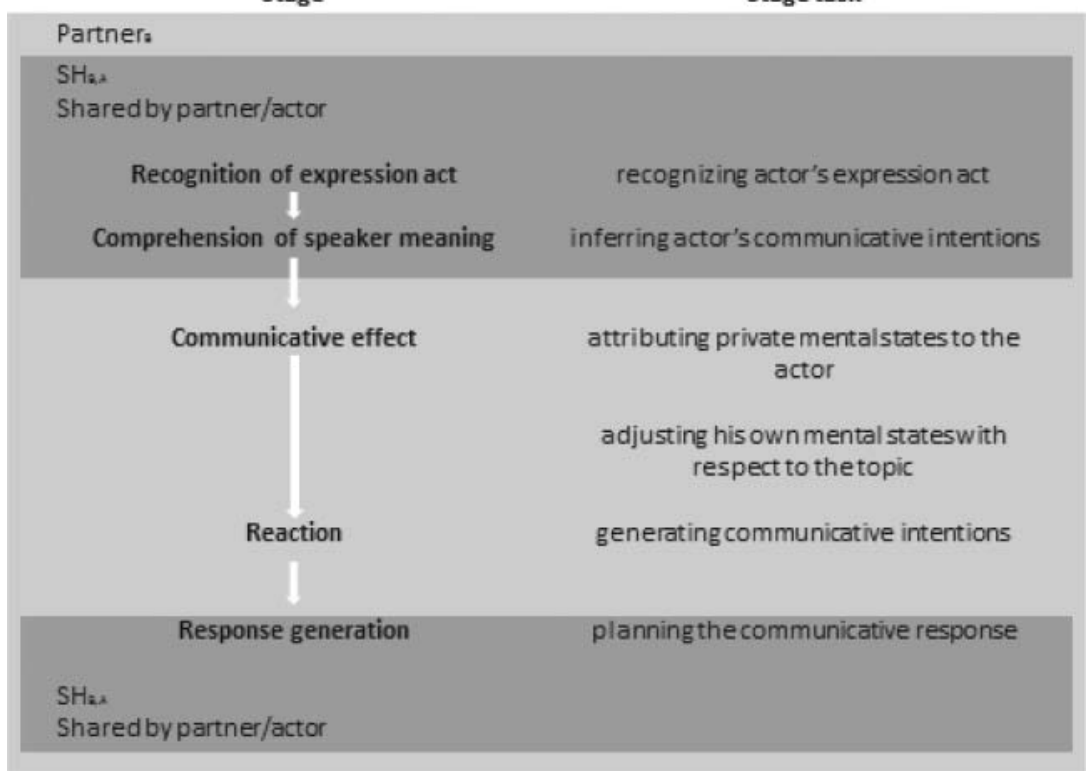

Figure 5. The five stages of comprehension and generation of a communication act.

Source: $\quad$ Airenti, Bara \& Colombetti 1993.

such rules are a convenient means for describing communicative interaction. This does not imply they actually exist in the mind or in the brain.

Comprehending the expression act (stage 1) and speaker meaning (stage 2) are managed by a limited number of specialized rules. The reason for this is that comprehension is a process that is shared and achieved by two people, with the result that the actor must, in principle, be able to predict how her partner will reconstruct the meaning of her utterance. Stated differently, since comprehension rules are constitutive of meaning, meaning's construction is shared by all those who are taking part in the interaction.

In contrast to the two initial stages, the effect of the utterance on the partner is a question of private mental processing. In this case, individual motivation and general intelligence prevail over shared social norms. This means that it is impossible to formulate an exhaustive set of rules for stage 3 .

Stage 4 , the reaction stage, is again different. The task here consists in planning a communication act whose starting point is the private motivations activated by the flow of the dialogue. It should therefore be possible to identify a set of norms that describe cooperative interaction. Such norms are neither universal nor logically necessary. They depend on the specific culture the agents belong to and on the specific circumstances the dialogue takes place in: In our 
terms, on the behavior game that is being played. This being the case, I do not introduce base-level response rules, but will simply present a few paradigmatic examples. The task of defining cultural and situational taxonomies of the rules for stage 4 must be left to the scholars of ethnicity.

Finally, response generation (stage 5) is based on a highly specialized type of planning and on a set of shared and constitutive linguistic and extralinguistic rules.

\subsection{Simplicity and complexity of a communication act}

I define a simple communication act as that in which the passage from utterance to the behavior game of which the utterance may be considered to be a move is immediate, requiring only one single inferential step.

I define a complex communication act as that in which the passage from utterance to the behavior game of which the utterance may be considered to be a move requires an inferential chain of variable length.

This generalization allows us to distinguish not only between simple and complex communication acts in standard communication (as in the case of indirect speech acts), but also between simple and complex communication acts in nonstandard communication, as in the cases of irony and deception.

4.1.1. Simple and complex indirect speech acts. The problem of recognizing indirect speech acts does not exist in cognitive pragmatics, since there is no primitive notion of nonliteral speech act. The key point for the partner is always that of recognizing the opening bid in a behavior game, in whatever form that move is expressed. Once the game has been identified, inferring what move the actor is asking her partner to make presents no extra difficulties whatsoever for indirect speech acts compared to direct speech acts.

In brief, the idea is that the locutionary act is always necessary as the starting point, but is not enough, even in those cases that are classically defined as direct speech acts. My expression act is de facto the unavoidable departure point for the reconstruction of speaker meaning, through the identification of a valid behavior game.

\section{Nonstandard communication}

The theory outlined in the preceding section describes the process of comprehension and of the construction of the response in situations that may be classified as standard, that is, situations that trigger default rules. There are, however, a large number of interesting cases that go beyond the bounds of normality, which may be classified under four headings: 
1. Nonexpressive interaction: the use of an utterance without there being any intention to express the mental state associated with that utterance.

2. Exploitation: the special use of a communication rule to obtain a communicative effect that is different from that normally associated with that rule (e.g., to create irony).

3. Deception: the attempt to convey a mental state that is not in fact possessed.

4. Failure: unsuccessful attempt to achieve the desired communicative effect.

The analysis of these cases is important not only in itself, but also because it provides independent evidence in favor of cognitive pragmatics, in the sense that the structural features typical of possible interactions that are nonexpressive, that exploit, that deceive, and that fail, overlap perfectly with the different representations on which the cognitive process of communication is based. An interruption in the standard inferential chain may be ascribed to one of two different reasons: either the actor wanted the interruption to come about, or else it comes about in the partner's mind without the actor's having wished it. Intentional interruption means that the actor intended to employ a form of exploitation or of nonexpressive interaction.

Alternatively, failure comes about either because the partner does not follow the inferential chain when he was meant to, or, conversely, because he follows the chain when he was not supposed to, since the actor had proposed a nonstandard mode. Finally, an attempt at deception takes place when the actor employs false shared knowledge in order to achieve her objectives.

As we saw in section 3.6, it is the conversation game that governs the succession of the five stages in communication. Stated differently, the conversation game works at a metalevel - employing metarules - that ensures that at the base level all the standard inferences may follow smoothly in succession without any blocks occurring, simply by applying the base rules. The purpose of the conversation game is, on the one hand, to guarantee that each stage accomplishes its task in an adequate fashion, thereby enabling the successive stage to receive the information it requires to proceed, and, on the other hand, to intervene if a given stage fails to achieve its objectives.

Indeed, if a stage does not realize its predetermined goals, then the conversation game intervenes at the metalevel in order to specify what has to be done about the problem that has occurred at the base level. In this case, the metalevel blocks the default rules that are specific to that stage, activating an alternative inferential process that does not employ that particular type of automatic rules. Just as I have termed the communicative process utilizing default rules standard, so I will call nonstandard those processes that must have recourse to classic inferencing procedures since they cannot apply standard default rules because the latter are inappropriate to the context. 
Each nonstandard situation has a logical place in the framework of the comprehension process I have outlined. Nonexpressive interaction, exploitation, and deception fall naturally into the first three stages of my model. In the first stage - understanding the expression act - the only nonstandard path the actor may have followed, and which the partner must therefore identify, is that of nonexpressive interaction. In the second stage - understanding speaker meaning - all nonstandard inferences are cases of exploitation. In both types of cases, the actor tries to ensure the partner will identify the nonstandard path and follow it correctly. Should this not happen, the outcome will be failure.

The third stage - communicative effect-is where deception occurs. Deception cannot be found in the comprehension stages because deception is not realized by any special form of communication. It concerns the relationship between what the actor communicates and her private mental states.

Finally, one has to analyze failures, which may occur at any stage. In order to recognize and possibly to repair a failure, one cannot use the default rules of communication: she has to fall back on classic inferential procedures.

In this paper I shall discuss only irony and deception, the two most interesting cases of nonstandard communication.

\subsection{Irony}

Let us suppose that B is telling A how his wife tried to use her car to knock down the woman whom he had decided to live with, and that A comments on the episode thus:

(11) "Your wife is definitely someone who does not take things badly!"

What makes utterance (11) ironic is the fact that both interlocutors share the view that the opposite of what was asserted is literally true. This blocks the application of the rule that lays down that, within the space of shared beliefs, if the actor expresses a belief, then by default she intends to communicate that this belief will be shared by herself and her partner, unless her partner denies the fact.

Example (11) illustrates the principal feature of exploitation: It is one way of playing with sharedness. In actual fact, utterance (11) could have been interpreted as a serious confirmation by a third person who had arrived after the initial description of the events furnished by B. The point is that an ironic intention does not alter the standard sequence in which the rules are applied, but only the way in which the rules are used. I have already stated that the rules are usually exploited by taking the reverse of the literal meaning conveyed by the utterance. This, for Grice (1989), is the essence of irony: saying $p$ and meaning non- $p(\sim p)$. For an extensive semantic and pragmatic analysis of irony, see Attardo (2001). 
Grice, of course, is formalizing the traditional analysis of irony: a trope based on antiphrasis, which is, quite simply, an utterance to be intended as its opposite. However, two kinds of argument have been advanced against equating irony exclusively with the opposite of literal meaning. The first was propounded by Sperber and Wilson (1995 [1986]), who demonstrated that ironic statements do not necessarily correspond to the opposite of literal meaning. Take the following excerpt from Voltaire's Candide (1947):

(12) When all was over and the rival kings were celebrating their victory with Te Deums in the respective camps....

According to Sperber and Wilson, irony is a case of echoic interpretation. An echoic utterance is one that echoes the thought, and the attitude toward the thought, of someone who is not the speaker herself. It echoes the thought of another person when it simply reports what that other person said. It may, however, echo traditional thoughts, as when it reports an obvious truth, a popular belief, or a proverb.

In the case of irony, the speaker produces an utterance that echoes another person's thought, while concurrently manifesting a critical or disparaging attitude toward content $p$. In example (12), Voltaire is not suggesting that neither of the two kings won the battle and were celebrating victory, nor that both kings lost the battle and were bewailing their defeat. Instead, Voltaire is echoing the claims made by the two rivals. Since the two simultaneous claims of victory contradict each other, it is obvious that both parties cannot be right. The irony stems from the fact that Voltaire highlighted the attitude of the rival kings, unveiling its vacuousness.

A second objection is advanced by Morgan (1990). He points out that no one-neither Grice, nor those such as Perrault (1990) who adopt Grice's approach — ever explains why a meaning $p$ should be interpreted as $\sim p$, and not as a lie. Morgan considers irony to be a transparent fiction: the speaker says something pretending to believe it while simultaneously making it obvious through the use of paralinguistic and nonverbal features that the utterance is indeed a fiction.

The vast majority of critics of the antiphrasis position are quite vehement in their censures. Morgan defines it as a perverse obscurity, while Sperber and Wilson declare that defining irony as implicating the opposite is a bizarre practice. As the criticisms leveled at the classical account demonstrate, Grice has solved one particular case, but not the general case: Sometimes in uttering $p$ the speaker implicates $\sim p$.

My theory is based essentially on shared knowledge, in order to explain how interlocutors understand - without the aid of the stigmatized miracles of the classical approach - that a given utterance is not to be taken literally. With 
regard to the meaning of irony, a more general explanation compared to those advanced so far is that the ironic utterance constructs a possible scenario that acts as a background against which the element of alterity introduced by the speaker without mentioning it, and which the hearer will have to infer, will be highlighted by contrast. Against this background, different meanings may emerge, including Gricean opposites, Sperber and Wilson's echoes, or Morgan's transparent fictions.

Thus, in (11), the possible scenario of a tolerant wife starkly foregrounds her attempt to kill her rival. In (12), the scenario, which is not merely possible but real, forcefully underscores how ridiculous the conflicting claims of the rival kings are. Even in the case of Buster Keaton's wordless irony, what the actor does is construct a possible scene that patently reveals the total incongruity between the actor's impassive behavior and the catastrophes occurring all around him. The element that represents alterity must emerge by contrast: white if the background is black, and black if the background is white.

Grice and those like Perrault who follow in the classical tradition focus essentially on the induction process the interlocutor must enact. However, they impose far too narrow confines, limiting it to the case, which is admittedly frequent, in which the emergent meaning is the opposite of the literal content of the utterance.

Sperber and Wilson concentrate on a particular type of scenario, one simulating a hypothetical agent, or one in which the speaker echoes thoughts of one or more agents. There is no doubt that this thesis furnishes a brilliant solution to some cases. However, not all cases of irony can be accounted for by echoic utterances. Turning to inductive processes, which Sperber and Wilson believe may be explained by the single principle of relevance, little attention is devoted to shared knowledge, which plays such a central part in the planning and comprehension of ironic utterances.

Let us now move on to a more formal presentation of my theory. Actor A generates utterance $p$, which is incompatible with state of things $r$. She also believes she shares knowledge $r$ with the addressee to whom the ironic utterance is directed, such knowledge having been adequately activated. Finally, her communicative intention is to contrast utterance $p$ with background $r$. Any spectators present may be divided into those who share belief $r$ with the actor and may therefore comprehend the ironic intent, and those who do not share belief $r$ and are therefore unable to understand what A really meant.

The general formula representing this is as follows:

(13) $\mathrm{BEL}_{\mathrm{A}} p \neq r$

$\mathrm{BEL}_{\mathrm{A}} \mathrm{SH}_{\mathrm{BA}} r$

EXPRESS $_{\mathrm{A}} p$

$\mathrm{CINT}_{\mathrm{A}} \mathrm{SH}_{\mathrm{BA}} p \neq r$ 
Recapitulating: since actor A is convinced that $p$ is incompatible with $r$, and thinks that belief $r$ is shared with $\mathrm{B}$, she utters $p$ ironically against the background of $r$.

All types of irony, antiphrasis included, fall within the scope of this general framework. All that is required is to recall the concepts of simplicity and complexity introduced at the beginning of this chapter. I will speak of simple irony when the interlocutor can grasp speaker meaning instantly, moving directly from the utterance to the behavior game of which the utterance may be considered to be a move. Example (11) is a prototypical instance of simplicity.

In more formal terms, if, for the sake of clarity, we concede for one moment that $r$ is the equivalent of non- $p$, A produces utterance $p$ that contrasts forcefully and immediately with shared belief $r$ (non- $p$ ).

I define complex irony as irony in which the interlocutor must carry out a series of inferences in order to grasp speaker meaning. This consists of an utterance $q$ that does not directly come into contrast with shared belief non- $p$. However, performing a series of inferences (from the utterance $q$ to its implication $p$ ) will enable the hearer to arrive at the conclusion that a belief $p$ is clearly incompatible with the activated scenario of shared beliefs. Example (12) is a prototypical case here.

In more formal terms, and still conceding momentarily for the sake of clarity that $r$ is the equivalent of non- $p$, A produces utterance $q$ from which $p$ may be inferred, and which manifestly and instantly contrasts with shared belief $r$ (non-p).

We can thus use one single analytical framework to account for extremely different types of irony, without having to forgo in the analysis either the infantile prank or the most sophisticated sarcasm. I have discussed only the linguistic type of irony, but the same treatment can also be extended to extralinguistic irony, such as clapping one's hands after somebody's poor performance at a trivial task.

\subsection{Deception}

The meaning of deception from an evolutionary standpoint is to be sought in the possibility of introducing into the struggle for survival and the reproduction of one's genes components that are not limited to brute physical force. A cogent case in point is that of the small seahorse, which manages to introduce himself into the dominant male's harem by pretending to be a female while the dominant male is too busy fighting off the official challengers. Despite their suggestiveness and the interest they arouse, these deceptions are limited since animals do not possess the mental capacities required to represent shared beliefs and communicative intentions, which means that they are unable to 
produce any form of communicative behavior, violations included, in the strict sense of the term.

For cognitive pragmatics, deception is a conscious violation of a shared behavior game. Though A knows she should act in a certain way in order to respect the behavior game being played by $\mathrm{B}$ and herself, she carries out a communicative behavioral act that is premeditated to make B believe it is a game move even while she knows full well it is a violation of that game.

I will now present the formulas necessary to represent deception in cognitive terms. Temporal notation is obligatory. However, such notation will be suppressed in the exploration of the full example for the sake of simplicity. The deceptive utterance takes place at time $t_{1}$; the beliefs and mental states preceding the utterance are indicated at time $t_{0}$; the progressive consequences of the utterance are indicated at times $t_{2}, t_{3} \ldots t_{n}$.

$$
\begin{array}{ll}
t_{0} & \mathrm{BEL}_{\mathrm{A}} \text { non- } p \\
t_{1} & \mathrm{CINT}_{\mathrm{A}} \mathrm{SH}_{\mathrm{BA}} p \\
t_{2} & \mathrm{EXPRESS}_{\mathrm{A}} p \\
t_{3} & \mathrm{BEL}_{\mathrm{A}} \mathrm{SH}_{\mathrm{BA}} p
\end{array}
$$

Summing up: A expresses utterance $p$, though not believing it herself, her communicative intention being that $\mathrm{B}$ take it as shared between them. If the deception works, A will assume that B takes $p$ as shared.

Stated differently, on the one hand, A hopes that B will believe $p$, and that he is convinced that A herself believes $p$ too; on the other hand, A commits herself to behaving for the rest of the interaction in a manner consistent with this "supposedly shared belief" (whereas B thinks it is shared, A does not believe it). From the standpoint of the cognitive resources required, the difficulty of deception consists in always keeping the private belief non- $p(\sim p)$ and the supposedly shared belief $p$ both active in her attentional space.

This latter type of nonstandard communicative situation revolves around the relationship between the mental states that actor A communicates and the private mental states she actually holds. As we have noted previously, the conversation game is neutral with regard to the sincerity and correctness of the interlocutors' behavior. Hence the participants may carry on a conversational dialogue that is correct from an external standpoint without ever communicating their mental states. For example, a promise may be expressed in an impeccable manner without there being any real intention to keep that promise. Indeed, from Delilah to Judas the best instances of treason have always been clothed in a form that aroused no suspicions whatsoever.

Not all acts of deceit have an equally complex structure. As for the other types of communication, the difficulty of an act of deception depends on the number of inferences B requires to reach the hidden game, starting from A's untruthful communication act. To carry out or discover a well-planned deceit, 
several aspects will have to be taken into consideration, in addition to the simple fact of whether $p$ is true or false. In principle, there is no limit to how complex a situation can be. Human beings are, however, incapable of dealing simultaneously with a high number of embeddings.

Employing the distinction between simple and complex speech acts once again enables us to establish a criterion by which to distinguish different levels of difficulty that a deceit may reach.

A simple deceit consists of the production of a communication act $(p)$ that contrasts with something (non- $p$ ) that would immediately allow the partner to identify what game the actor is trying to hide from him. Simple deceit, as I have defined it, is called lying in the literature.

A complex deceit consists in producing a communication act $q$, implying a belief $(p)$ that leads the partner toward a move or a game which is different from that he would arrive at if he had access to A's private belief non- $p$.

Thus, lies and deceits are not always conceptually distinct. They fall along a continuum in which the lie constitutes the simplest level. The more complex the deceit, the greater the planning it requires. But even the most intricate deceit sometimes requires the telling of a lie, by which I mean an untruth. In this latter case, the lie is only the ultimate act finalizing a planning process that is potentially extremely complex.

In order to succeed, the deceit must not be discovered by the partner. If the partner discovers an attempt to deceive him, he may declare the fact or he may pretend he has not realized, in order to plan a counterdeceit, if he so wishes. The possibility of there being a counterdeceit demonstrates that A may simulate B's mental processes only up to a certain point, and in any case the operation always involves a certain amount of risk.

Finally, deceits also may be produced through extralinguistic means, from pointing to a wrong location (simple deceit) to taking off one's wedding ring before leaving for a conference out of town (complex deceit).

\subsection{Failure}

Even though it might at first seem paradoxical, from the standpoint of cooperation, even a failure must be agreed on by the actors. In order to remain consistent with all the literature on the subject, I will continue to use the term failure in a generic sense to indicate all those cases that exhibit communicative failure at whatever level this might have taken place. I will introduce the term shared failure to indicate that particular case in which both the agents are aware of what has happened and accept the definition of the occurrence as constituting one of failure in the proper sense of the term. What I wish to underline is the fact that to define an occurrence as a shared failure both the agents must consciously recognize that there has been a nonnegotiable refusal, and that this 
refusal is to be considered as shared knowledge. If one of the two agents, either A or B, does not believe that this is what has happened, then the case cannot be classified as shared failure, despite the difficulties that have occurred.

We are now in a position to define a communicative failure as an abortive attempt to produce a given communicative effect on one's partner. From A's standpoint, communicative failure may come about in any of the first three stages I have hypothesized: at the level of the expression act, at the level of speaker meaning, or at the level of communicative effect. An interruption at any point along the communicative chain will produce a failure to achieve the speaker's goal, that is, the speaker will fail to generate in her partner's mind the mental state she intended to generate.

There are three types of communicative failure: noncomprehension, misunderstanding, and refusal. I discuss these in turn.

5.3.1. Noncomprehension. B fails to understand the expression act or the speaker meaning of what A is saying. An interruption in the inferential chain is detected at the level of the conversational game. Since the predefined task of the pertinent metarule has not been completed, the regular flow of the successive stages is interrupted and the reaction stage is activated. In the reaction stage the partner decides whether to render the failure explicit, for instance by asking for clarification, or whether to handle the situation in a different way. Whatever option he does adopt, failure is transparent to him, and his choice of option is a conscious one. Noncomprehension is a case of transparent failure for $\mathrm{B}$, for $\mathrm{B}$ is aware he has not understood what A was saying.

5.3.2. Misunderstanding. B fails to comprehend the expression act or the speaker meaning of what A is saying in the way she intended it to be interpreted. The inferential chain takes a different route from the one A intended it to take. Misunderstanding is a case of opaque failure from B's standpoint, in the sense that B is unaware that he has failed to understand what A has said.

5.3.3. Refusal. B understands what A is saying, but refuses to comply with A's orientation. In this case, the private mental states of B are involved, either in the attribution process (inferring private mental states of A), or in the adjustment process (modifying B's own mental states), something followed a different route from the one $\mathrm{A}$ wished it to take. Refusal is a case of transparent failure for B, in the sense that it depends on a conscious decision on B's part.

5.3.4. The developmental approach to failures. The study of failure is crucial from a methodological point of view, because it allows us to observe the intermediate erroneous outcomes of the communication process. A theory able 
to predict errors that occur during a process is to be praised over theories that can predict only correct responses.

None of the existing pragmatic theories offers a global account of successful and failed communication. A noteworthy exception is relevance theory, which establishes a continuum between the idealizations of success and failure; Sperber and Wilson (1995 [1986]) measure the efficacy of communication in terms of attempted relevance, as compared with achieved relevance. The introduction of strong and weak implicatures emphasizes the idea that each communicative instance conveys core meaning and perpetual implications. The notion of failure is spread over a wide set of implicatures, both those attempted and those that can be possibly achieved. Still, relevance theory has never generated systematic hypotheses for explaining communicative failures.

Bosco, Bucciarelli, and Bara (2006) proposed a taxonomy of different sorts of failure, which is grounded on the mental representations and cognitive processes involved: failure of the expression act, failure of the speaker meaning, and failure of the communicative effect. When failure of the expression act occurs, the partner fails to comprehend the literal value of the utterance; when failure of the speaker's meaning occurs, the partners fails to comprehend the speaker's communicative intention; and finally, when failure of the communicative effect occurs, the partner does not modify his mental states in the way the speaker desires, that is, he refuses to adhere to the speaker's goal. Depending on the sort of failure occurred, the speaker might enact a different kind of repair. The taxonomy allows us to generate hypotheses about the relative difficulty in recognizing and repairing different kinds of failure.

Our account follows a developmental perspective. This means studying mental processes not only as fixed states - an approach that takes into consideration exclusively the final stage - but rather concentrating on how a given function develops in the infant to the child and the adult. In particular, we studied children aged 3 to 8 years.

Globally considered, the results of the experiment support our taxonomy and its underlying assumptions. A first result concerns the distinction between the recognition of successful communication acts and the recognition (and subsequent repair) of the respective failures. It is simpler to recognize the success of a communication act than the respective failure. Thus, children perform better at recognizing that the speaker has succeeded in modifying the partner's mental state in the desired way, than at recognizing when the speaker has failed in her attempt. The hypothesis holds for all kinds of failure and supports the assumption that inconsistencies between A's private goal and its felicitous realization are hard to detect.

As far as recognition of failures is concerned, noncomprehension is easier to recognize than misunderstanding, and failure of the expression act is easier to recognize than failure of the speaker meaning. Failure of the communicative 
effect is quite easy to recognize, and as predicted it posits at the same level of difficulty as failure of the expression act. Also, as we obviously could expect, it is simpler to recognize the failure of a communication act than to recover such a failure. One does not repair something if she does not think it failed.

\section{Communicative competence}

Bypassing the controversial issue of the evolution of communication in the last 4 million years (from Australopithecus on), I will focus to its development from childhood in anatomically modern Homo sapiens (last 200,000 years). The phase we are living in now began only 35,000 years ago, when the union of the potential for language and for external cognition gave rise to permanent linguistic structures, generating writing, which is in its turn allowed history.

\subsection{Linguistic and extralinguistic writing}

Humanity's most brilliant invention consists in using our capacity to draw symbols to ensure the preservation of knowledge at the group level, that is, beyond the life of the single individual. The invention of writing enables external cognition to become permanent, thus making transgenerational evolution possible. Stated differently, the conditions necessary for the existence of culture, intended as the possibility to transmit knowledge acquired individually to other members of the group, are external cognition and its consolidation, that is, its relative permanence.

A full reconstruction of the evolution of writing is in Bara 2003, which I here summarize. The first step toward writing is the moment when the first human artists begin to paint and sculpt cave walls. In a continuous evolution lasting at least 40,000 years, aesthetic, magic, and sacred functions are complemented by those conveying cognitive content. We may state that the earliest traces of graphological symbols go back 35,000 years, and consist of small, equidistant engravings whose significance is still incomprehensible, but whose function may be stated as being that of acting as an external support for an individual's memory, or perhaps for that of a group.

Returning to the distinction between linguistic and extralinguistic communication, and given the fact that the feature of permanence may be attributed to both modes, we should be able to recognize an extralinguistic mode of writing as well as the linguistic mode that we are used to. Alexander Marshack (1991) has documented what is still today the first instantiation of protowriting. This goes back to the Paleolithic age, around 10,000 B.C.E. A bone fragment was discovered in Tai, France, bearing mathematical notation as well as writing. 
Presumably this was allotted to marking the passage of time - a sort of elementary calendar.

We may distinguish between the various forms of writing by using the number of signs employed as our classificatory criterion: thus pictograms employ over a 1,000 signs, logograms employ between 1,000 and 40 signs, and an alphabet uses fewer than 40 signs.

The most ancient example of pictogram is the calcareous slab found in the Sumerian city of Kish in Mesopotamia. The drawing of a foot, a hand, and a sleigh, beside which are symbols that are presumably numbers, appears for the first time around 3,500 B.C.E. The clay tablets found inside the temple in the city of Uruk in Mesopotamia exhibit 1,500 different symbols. Each of these pictograms refers to an object: The head of a bull represents a bull, the outline of a mountain a mountain.

The efficacy of the pictogram simultaneously constitutes its limit: Everyone can immediately grasp the meaning of the signs drawn on the tablet, seen as a sequence of images. It constitutes a kind of representation by association: Its true meaning can only be comprehended by those who are already familiar with facts represented before their eyes. Pictography leaves aside the times, modes, and logical links that connect subjects and events. This is why I classify pictography as a permanent version of extralinguistic communication: a set of symbols having associative, noncompositional structure. I will now examine the transition stage to the permanent representation of linguistic communication.

To increase the effectiveness of pictographic writing, signs must be stylized, which reduces the number of signs that may be used. This is what happens to logograms. The Egyptians, who needed an efficient system of writing in order to rule their large empire, solved the problem around 3,000 B.C.E. What the eye is about to perceive becomes schematized. Thus a pubic triangle is used to represent a woman, an eye to represent seeing, and so forth. But the crucial step consists in introducing a rudimentary form of syntax, which allows the communicators to move from the property of association, which characterizes extralinguistic communication, to that of compositionality, the feature that defines linguistic communication.

Two of the most important indicators of the inception of the principle of compositionality are the plural form and the combination of logograms. Two signs of the same type indicate the plural: the sign for a bird indicates a bird, two signs of a bird indicate birds in the plural. It is also possible to create new ideograms by combining two or more different signs: For instance, mouth + bread $=$ eat; woman + mountain $=$ serve; woman + dress $=$ mistress of the house. It should be noted that ideograms are a direct reflection of the meaning of the sign - there is no link with the way the word is pronounced. 
The final crucial step toward writing as we know it today is the substitution of ideograms by phonograms, which comes about in Mesopotamia around 2,500 B.C.E. This reduces the signs employed to fewer than 100 . The main mechanism that realizes this operation is a crossword-puzzle-type principle, a principle related to the idea of rebus. For instance, in order to represent the concept of a date, the writer does not use a symbolic representation of the calendar (a sun followed by a moon). Instead he draws a date (the fruit). What creates the form-meaning link is not the sign in itself, but the uttering of the sign. We have thus reached the stage of syllabic writing, such as the systems in Mesopotamia and Egypt, which are no longer simply looked at as were pictograms, and are not yet read as are alphabet systems: These are deciphered.

The final step consists in the invention of the alphabet by the Phoenicians, around 1,700 B.C.E. This time the pressure for change came from trade, not from conquest, as was the case with the Egyptians. The Phoenician alphabet had 22 signs; it used only consonants, leaving the reader with the task of adding the vowels. Vowels were introduced into the alphabet by the Greeks around 1,000 B.C.E., thereby achieving total correspondence between spoken and written linguistic communication.

\subsection{Developmental pragmatics}

The human adaptation for symbolic communication emerges in human ontogeny quite steadily at around 1 year of age (Tomasello 1995; Liebal et al. 2009) in the context of a whole range of new social-cognitive skills, which may be referred to as skills of intention-reading (i.e., establishment of joint attentional frames, understanding communicative intentions and so on). Language production emerges gradually in children in the months following the first birthday just because they need that these fundamental skills of intentionreading are firmly in place (Tomasello 2003). Children's symbolic productions are not confined only to language, but they include various kinds of gestures, from simple ritualizations to symbolic gestures, demonstrating that words and gestures are all a part of the same developmental communicative process.

The investigation of children's communicative acquisition has been mostly conducted on the earlier stages of development. However, children are continuously dealing with various kinds of communication acts, from simple to complex, during all childhood years, learning how to communicate more effectively in extended conversational exchanges with other people. My colleagues and I investigate the children's linguistic and extralinguistic progress within a coherent developmental perspective. Our work has two main ambitions: 
1. To study in parallel the emergence of linguistic and extralinguistic communication, reunified under a single theoretical paradigm.

2. To offer a baseline against which to compare atypical performances in relation to linguistic and extralinguistic conditions.

Children with atypical development may show different deficits in the comprehension and production of communication acts, depending on the type of pathology, namely head injuries, hydrocephalus, focal brain damage, and autism (Bara, Bosco, and Bucciarelli 1999). But the key point is that it is hard to understand the nature of the deficits when one does not know how normal development takes place. Our approach aims to clarify the factors involved in the comprehension and production of the different kinds of communication acts, following their emergence during typical development in order to deeper understand their deficits in case of pathological conditions.

Three factors determine the complexity of the mental representation involved in the comprehension of a pragmatic phenomenon (see Figure 6). The factors involved may be referred as (1) conflicting representations, (2) exploitation of sharedness, and (3) inferential load (Bucciarelli, Colle, and Bara 2003).

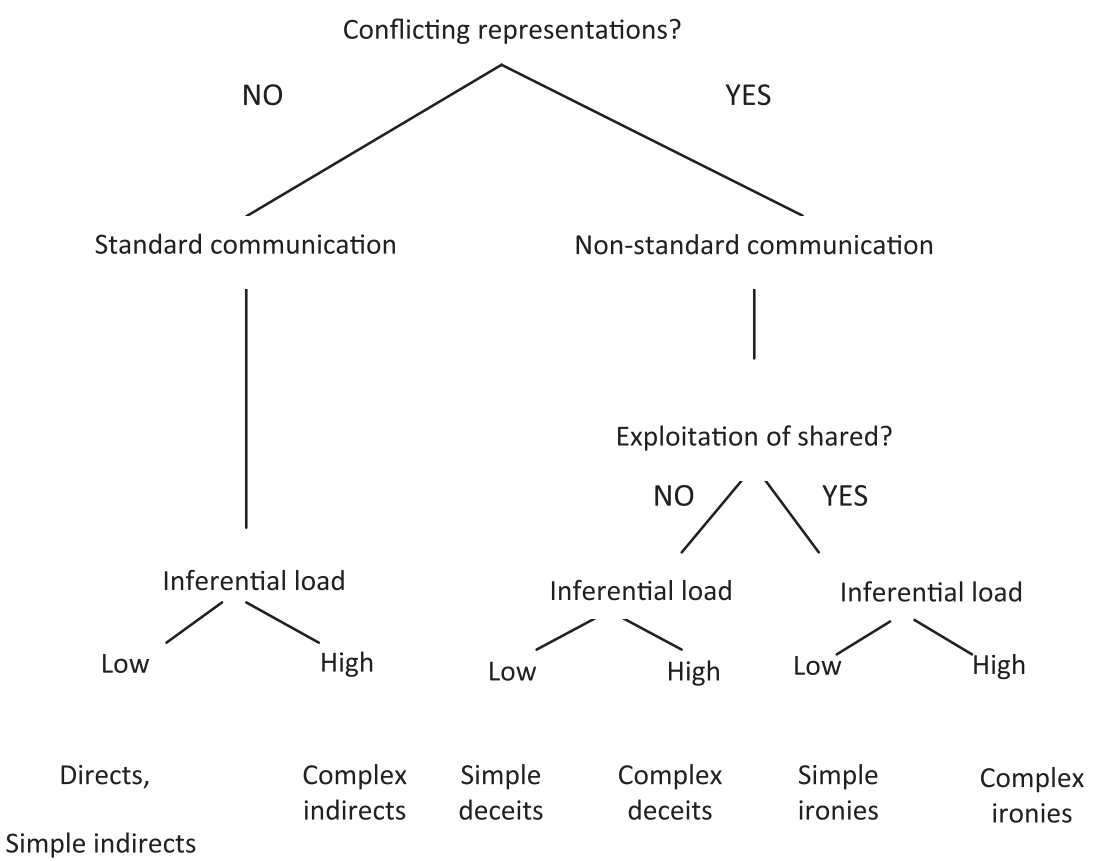

Figure 6. Factors that determine the difficulty of comprehension of pragmatic phenomena. 
6.2.1. Conflicting representations. Conflicting representations involve a discrepancy between what is communicated and what is privately entertained by the actor. In the case of no conflict, we are dealing with standard communication, where the actor merely produces an utterance that is in line with her private belief and with the behavioral game she shares with the partner. In the case of conflict, we are dealing with nonstandard communication, which involves the violation of default rules and the occurrence of more sophisticated mental representations.

Direct communication acts, conventional indirects, and nonconventional indirects are all examples of standard communication: In terms of mental representations, the partner has merely to compare the actor's utterance to a valid behavior game. Deceit and irony are instead examples of nonstandard communication, in which the partner has to deal with the difference between the mental states expressed by the actor and those she privately entertains. It may be consequently predicted that standard communicative phenomena are easier to deal with than nonstandard ones.

6.2.2. Representations where shared beliefs are exploited. Representations involving a belief expressed by an actor that is in contrast with a belief shared with the partner are more difficult to handle than representations that do not involve such a contrast.

When comprehending a deceit, the partner recognizes the discrepancy between the mental states that are expressed and those that are privately entertained by the actor. An utterance becomes ironic when, along with this discrepancy, the partner also has to recognize the contrast between the expressed mental states and the scenario provided by the knowledge shared with the actor. The concurrent activation of the representation of actor's utterance $(p)$ and of the contrasting shared belief makes ironic statements difficult for a child to manage. It follows that deceits should be easier to deal with than ironies.

6.2.3. Inferential load. The necessity of building a long chain of inferences is what discriminates between simple and complex communication acts. Direct and conventional indirect speech acts may be referred as simple communication acts because they immediately make reference to a behavioral game. Nonconventional indirect speech acts may be linked to complex communication acts because they do not make direct reference to a behavioral game and require a more complex inferential process. The length of the inferential chain necessary to connect the communication act to the behavioral game shared between the interlocutors is the factor determining the different levels of difficulty children find in comprehending simple and complex communication acts. 
In Bucciarelli, Colle, and Bara (2003) we started to investigate those theoretical points. We administered to a large group of children - ranging in age from 2.5 to 6 years old - an experimental protocol structured in both linguistic and extralinguistic modalities and comprising simple and complex pragmatic phenomena. Half of the children were randomly assigned to the linguistic protocol, and half to the extralinguistic one. The results of the study globally confirmed our predictions. Standard communication was easier to comprehend than nonstandard communication in both linguistic and extralinguistic modality. Also, in both modalities, directs and simple indirects were equally easy to comprehend, and they were easier than simple deceits, which were easier than simple ironies.

More recently, in order to provide a more comprehensive picture of children's pragmatic abilities, we used a new experimental protocol (see section 6.3) derived from the Assessment Battery for Communication ( $\mathrm{ABaCo}$, Sacco et al. 2008). The protocol was administered to 390 children ranging in age from 5 to 8.5 years old, and comprised a wide range of pragmatic phenomena (Bosco, Angeleri, Colle, Sacco, and Bara, under review).

In this study, we investigated different pragmatic phenomena (standard deceit and irony), different levels of complexity (simple and complex communication acts), different expressive modalities (linguistic and extralinguistic), and different perspectives (comprehension and production). The results confirmed again our theoretical predictions: First of all, in regard to the comprehension and production of standard and nonstandard communication acts, children's performances followed the same pattern in both the linguistic and extralinguistic modality, thus supporting the assumption that linguistic and extralinguistic communication share relevant cognitive factors and may be thought as different aspects of a single communicative competence.

Secondly, in regard to the variation among different pragmatic phenomena, we found that the difficulty in manipulating mental representations of increasing complexity also played a role in influencing children's pragmatic performance. The result is in line with our expectations: We found that children both understood and produced standard communication acts more accurately than deceit, followed by irony, which they understood and produced least accurately, both in linguistic and extralinguistic modality production.

Finally, the results concerning the comprehension of simple vs. complex communication acts extend also to the extralinguistic modality the idea that the increasing inferential chain required by complex communications acts is a relevant factor that may influence children's communicative performance.

The experimental results are summarized in Figure 7 and Figure 8. 


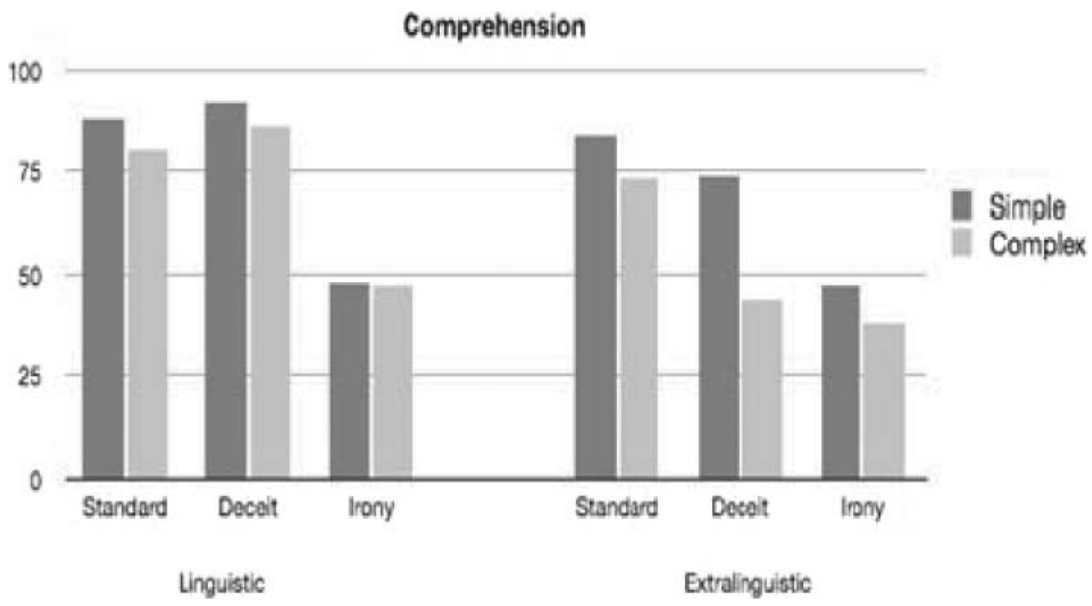

Figure 7. Comprehension. Children's comprehension of standard communication acts, deceit and irony, both for linguistic and extralinguistic modality.

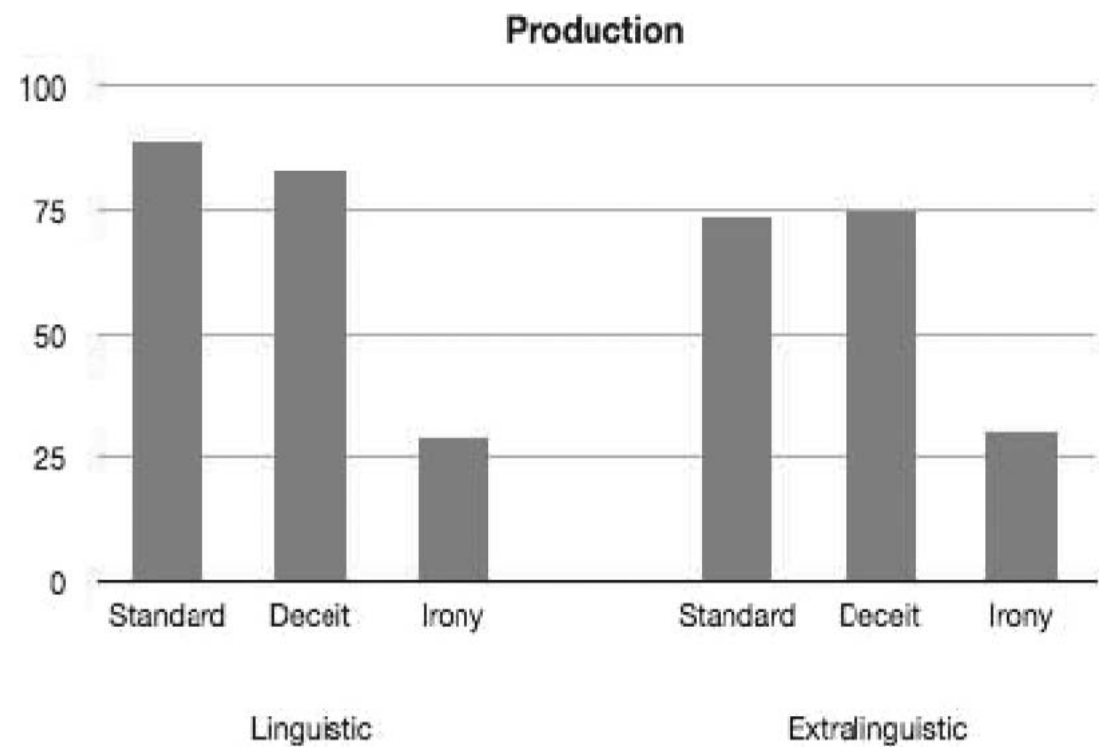

Figure 8. Production. Children's production of standard communication acts, deceit and irony, both linguistic and extralinguistic scale. 


\subsection{Neuropragmatics}

The aim of using a neologism in the title of this section is to draw attention to an area that has not yet been consolidated, but which is of great methodological importance: that dominion that investigates the correlations between the mental processes involved in communication and the areas of the brain that are responsible for those processes (Bambini and Bara 2011). In particular, in view of the fact that research in this sector is still in its initial stages, the data that will be examined are those that link up different types of cerebral lesions, both diffuse and focal, with particular impairments in communication (Bara and Tirassa 2000).

Taking up one of my initial methodological assumptions, namely that the mind is a biological structure, I will take it for granted that a valid theory of communication should be able to identify which areas of the brain underlie the physical realization of communication. This means specifying which areas of the brain are activated when a person is engaged in communicative activity (see section 2.3), and to look at the issue from the opposite viewpoint, what types of deficits may be predicted as a result of lesions occurring to any of these areas.

With different collaborators, I have carried out some research in this direction. Most of the work, however, remains to be done: We have only been able to test a few classes of subjects, such as individuals affected by traumatic head injuries (Bara, Tirassa, and Zettin 1997; Bara, Cutica, and Tirassa 2001; Cutica, Bucciarelli, and Bara 2006), patients suffering from Alzheimer's disease (Bara, Bucciarelli, and Geminiani 1999), neuropsychologically atypical children (Bara, Bosco, and Bucciarelli 1999), and autistic children (Bucciarelli, Colle, and Bara 2003). The aim of our experimental studies was first to outline for each type of patient a performance profile as regards the prominent pragmatic phenomena. The second aim was to explore in detail how each type of neuropsychological damage actually affects communication. Basically, in these studies we have found a trend analogous to that reported above in Figure 6.

The assessment of pragmatic abilities in patients affected by acquired cerebral lesions is relevant, since a deeper understanding of their specific communicative deficits will be crucial for both theoretical advances and clinical suggestions. Traditionally, communicative deficits have been interpreted as linguistic disabilities, but in more recent years clinical researchers have highlighted the need for a more comprehensive assessment that will offer a complete picture of patients' communicative effectiveness in their everyday life (e.g., Holland 1991; Penn 1999).

Our research group has recently created a new assessment battery, $\mathrm{ABaCo}$ (Assessment Battery for Communication), that can be profitably used to evaluate the various abilities involved in communication, comprising a wide range of pragmatic phenomena and communicative modalities (Sacco et al. 2008). 
$\mathrm{ABaCo}$ comprises five evaluation scales - linguistic, extralinguistic, paralinguistic, context, and conversational-which include several kinds of pragmatic phenomena - for example, deceit, irony, social appropriateness, Grice's maxims - for a total of 190 items. The idea was to create a new clinical tool, which would represent a valuable starting point for a complete understanding of the pragmatic deficits involved in different types of cerebral lesions.

We used $\mathrm{ABaCo}$ in a study aimed at investigating the pragmatic performances of traumatic brain injury (TBI) patients (Angeleri et al., 2008). Results showed that although patients' comprehension was damaged, it was nevertheless linguistically valid; on the other hand, they performed worse than controls in extralinguistic comprehension of deceit and irony. TBI patients showed impaired production in both the linguistic and extralinguistic modality. Moreover, the difficulty in manipulating mental representations had a great impact on patients' performance: we found an increasing trend of difficulty in different kind of pragmatic phenomena that require to deal with embedded mental representations. Specifically, both comprehension and production of standard communication acts were easier than deceits, which in turn were easier than ironies.

TBI patients also showed a pronounced impairment in managing paralinguistic aspects, neglecting the emotional meaning expressed through facial expression or prosody. Finally we found that TBI patients were impaired in grasping subtler conversational violations.

This study represents the first detailed investigation of pragmatic abilities in TBI patients, and shows how the cognitive pragmatics theory can be used to formulate specific predictions about the communicative impairments resulting from pathology.

\section{Conclusions}

In this paper I synthesized the main theoretical assumptions of cognitive pragmatics theory, as well as the empirical evidence enforcing the deriving predictions. Our experimental results and those in the literature on the development, the decay and the neural basis of communication, can be easily accommodated within the advanced unified framework.

\section{Acknowledgments}

This research was supported by Regione Piemonte, Project: Institutions, Behaviour and Markets in Local and Global Settings (Project IIINBEMA). 
I thank the friends that generously offered their time in order to help me: Mauro Adenzato, Romina Angeleri, Monica Bucciarelli, Nicoletta Causi, Claudia Chiavarino, Angela Ciaramidaro, Ivan Enrici, and Wendelin Reich.

\section{References}

Airenti, Gabriella, Bruno G. Bara, \& Colombetti Marco. 1993. Conversation and behavior games in the pragmatics of dialogue. Cognitive Science 17(2). 197-256.

Angeleri, Romina, Francesca M. Bosco, Marina Zettin, Kristina Sacco, Livia Colle, \& Bruno G. Bara. 2008. Communicative impairment in traumatic brain injury: A complete pragmatic assessment. Brain and Language 107. 229-245.

Attardo, Salvatore. 2001. Humorous texts. Berlin: Mouton de Gruyter.

Austin, John Langshaw. 1962. How to do things with words. Oxford: Oxford University Press.

Bambini, Valentina \& Bruno G. Bara. 2011. Neuropragmatics. In Jef. Verschueren \& Jan-Ola Östman (eds.), Handbook of Pragmatics. Amsterdam: John Benjamins.

Bara, Bruno G. 2003. Il sogno della permanenza: l'evoluzione della scrittura e del numero. Turin: Bollati Boringhieri.

Bara, Bruno G., Francesca M. Bosco, \& Monica Bucciarelli. 1999. Developmental pragmatics in normal and abnormal children. Brain and Language 68. 507-528.

Bara, Bruno G., Monica Bucciarelli, \& Giuliano C. Geminiani. 1999. The development and decay of extra-linguistic communication. Brain and Cognition 43. 1-3.

Bara, Bruno G., Angela Ciaramidaro, Henrik Walter, \& Mauro Adenzato. 2011. Intentional minds: A philosophical analysis of intention tested through fMRI experiments involving people with schizophrenia, people with autism, and healthy individuals. Frontiers in Human Neuroscience $5(7)$.

Bara, Bruno G., Ilaria Cutica, \& Maurizio Tirassa. 2001. Neuropragmatics: Extralinguistic communication after closed head injuries. Brain and Language 77. 72-94.

Bara, Bruno G. \& Maurizio Tirassa. 2000. Neuropragmatics: Brain and communication. Brain and Language 71. 10-14.

Bara, Bruno G. \& Maurizio Tirassa. 2010. A mentalist framework for linguistic and extralinguistic communication. Linguistic and Philosophical Investigations 9. 182-193.

Bara, Bruno G., Maurizio Tirassa, \& Marina Zettin. 1997. Neuropragmatics: Neuropsychological constrains on formal theories of dialogue. Brain and Language 59. 7-49.

Barbieri, Filippo, Antimo Buonocore, Riccardo Della Volta, \& Maurizio Gentilucci. 2009. How symbolic gestures and words interact with each other. Brain and Language 110. 1-11.

Berry, John W., Ype H. Poortinga, \& Janak Pandey. 1997. Handbook of cross-cultural psychology. Boston: Allyn \& Bacon.

Bosco, Francesca M., Romina Angeleri, Livia Colle, Katiuscia Sacco, \& Bruno G. Bara. Forthcoming. Communicative abilities in children: An assessment through different phenomena and expressive means.

Bosco, Francesca M., Monica Bucciarelli, \& Bruno G. Bara. 2006. Recognition and repair of communicative failures: A developmental perspective. Journal of Pragmatics 38(9). 1398-1429.

Bratman, Michael E. 1987. Intention, plans and practical reason. Cambridge MA: Cambridge University Press.

Bucciarelli, Monica, Livia Colle, \& Bruno G. Bara. 2003. How children comprehend speech acts and communicative gestures. Journal of Pragmatics 35(2). 207-241.

Chomsky, Noam. 1957. Syntactic structure. The Hague: Mouton. 
Ciaramidaro, Angela, Mauro Adenzato, Ivan Enrici, Susanne Erk, Lorenzo Pia, Bruno G. Bara, \& Henrik Walter. 2007. The intentional network: How the brain reads varieties of intentions. Neuropsychologia 45. 3105-3113.

Clark, Herbert H. 1996. Using Language. Cambridge: Cambridge University Press.

Cutica, Ilaria, Monica Bucciarelli, \& Bruno G. Bara. 2006. Neuropragmatics: Extralinguistic pragmatic ability is better preserved in left-hemisphere-damaged patients than in right-hemispheredamaged patients. Brain and Language 98. 12-25.

Duranti, Alessandro. 1997. Linguistic anthropology. Cambridge: Cambridge University Press.

Enrici, Ivan, Mauro Adenzato, Stefano Cappa, Bruno G. Bara, \& Marco Tettamanti. 2011. Intention processing in communication: A common brain network for language and gestures. Journal of Cognitive Neuroscience 23. 2415-2431.

Grice, Herbert Paul. 1989 [1975]. Logic and conversation: The William James lectures. 2nd edn. In Herbert Paul Grice (ed), Studies in the way of words, 22-40. Cambridge MA: Harvard University Press.

Grice, Herbert Paul. 1989. Studies in the way of words. Cambridge MA: Harvard University Press.

Gunter, Thomas C. \& Patric Bach. 2004. Communicating hands: ERPs elicited by meaningful symbolic hand postures. Neuroscience Letters 372. 52-56.

Harman, Gilbert. 1977. Review of "Linguistic Behavior" by Jonathan Bennett. Language 53. 417-424.

Hinde, Robert A. 1972. Non-verbal Communication. Cambridge: Cambridge University Press.

Hintikka, Jaakko. 1962. Knowledge and Belief. Ithaca, NY: Cornell University Press.

Hintikka, Jaakko. 1966. Knowing oneself and other problems in epistemic logic. Theoria 32. 1-13.

Holland, Audrey. 1991. Pragmatic aspects of intervention in aphasia. Journal of Neurolinguistics 6. 197-211.

Liebal, Kristin, Tanya Behne, Malinda Carpenter, \& Michael Tomasello. 2009. Infants use shared experience to interpret pointing gestures. Developmental Science 12. 264-271.

Marshack, Alexander. 1991. The Tai plaque and calendrical notation in the Upper Paleolithic. Cambridge Archeological Journal 1(1). 25-61.

Morgan, Jerry. 1990. Comments on Jones and on Perrault. In Philip R. Cohen, Jerry Morgan, \& Martha Pollack (eds.), Intention in communication, 189-193. Cambridge MA: MIT Press.

Pacherie, Elisabeth \& Patrick Haggard. 2010. What are Intentions? In Lynn Nadel \& Walter Sinnott-Armstrong (eds.), Benjamin Libet and Agency, 70-84. Oxford: Oxford University Press.

Penn, Claire. 1999. Pragmatic assessment and therapy for persons with brain damage: What have clinicians gleaned in two decades? Brain and Language 68. 535-552.

Perrault, Raymond C. 1990. An application of default to speech act theory. In Philip R. Cohen, Jerry Morgan \& Martha Pollack (eds.), Intention in communication, 161-185. Cambridge, MA: MIT Press.

Piller, Ingrid. 2010. Intercultural communication: A critical introduction. Edinburgh: Edinburgh University Press.

Popper, Karl R. 1934. Logik der Forschung. Vienna: Julius Springer Verlag.

Sacco, K., R. Angeleri, F. M. Bosco, L. Colle, D. Mate, B. G. \& Bara. 2008. Assessment Battery for Communication-ABaCo: A new instrument for the evaluation of pragmatic abilities. Journal of Cognitive Science 9. 111-157.

Searle, John R. 1983. Intentionality. Cambridge: Cambridge University Press.

Sperber, Dan \& Deirdre Wilson. 1995 [1986]. Relevance: Communication and Cognition, 2nd edn. Oxford: Blackwell.

Tomasello, Michael. 1995. Joint attention as social cognition. In Chris Moore and Philip J. Dunham (eds.), Joint attention: Its origins and role in development. Hillsdale, NJ: Erlbaum.

Tomasello, Michael. 2003. Constructing a language. A usage-based theory of language acquisition. Cambridge MA: Harvard University Press. 
Tomasello, Michael. 2009. Why we cooperate. Cambridge MA: MIT Press.

Voltaire. 1947 [1759]. Candide ou l'optimisme [Candide]. London: Penguin.

Walter, Henrik, Angela Ciaramidaro, Mauro Adenzato, Nenad Vasic, Rita B. Ardito, Susanne Erk, \& Bruno G. Bara. 2009. Dysfunction of the social brain in schizophrenia is modulated by intention type: An fMRI study. Social Cognitive and Affective Neuroscience 4(2). 166-176.

Corresponding address: bruno.bara@unito.it 